\title{
Slow and steady wins the race
}

\author{
Les James ${ }^{1}$ and Eugene Grossi ${ }^{1}$ \\ ${ }^{1}$ NYU Grossman School of Medicine
}

November 5, 2021

COMMENTARY: London Bridge is falling down - how will we build it up? AUTHORS:

Les James, MD, MPH General/Thoracic Surgery Resident Department of Cardiothoracic Surgery NYU Grossman School of Medicine Email: Leslie.James@nyulangone.org

Eugene A. Grossi, MD Stephen B. Colvin Professor of Cardiothoracic Surgery Director, Cardiac Surgery Research Department of Cardiothoracic Surgery NYU Grossman School of Medicine Email: Eugene.Grossi@nyulangone.org

\section{CORRESPONDING AUTHOR:}

Eugene A. Grossi, MD Stephen B. Colvin Professor of Cardiothoracic Surgery Director, Cardiac Surgery Research Department of Cardiothoracic Surgery NYU Grossman School of Medicine Email: Eugene.Grossi@nyulangone.org

CONFLICT OF INTEREST STATEMENT: Dr. Eugene A. Grossi has intellectual property and receives royalties from Medtronic for valve repair devices, and has intellectual property and receives royalties from Edwards Lifesciences.

ARTICLE WORD COUNT: 253 words (excluding references, tables, and legends)

COMMENTARY: Slow and steady wins the race - Building a Robotic Mitral Valve Program

This review of 7 years of a single center's experience with robotic mitral valve repair (RMVr) by Klepper and colleagues is a testament to the importance of dogged persistence in the pursuit of exceptional outcomes. Multiple studies have demonstrated that it is possible to achieve comparable results with RMVr, even in complex disease.[1, 2] However, widespread adoption of RMVr has been discouraged by some owing to reports of longer operative times, steep learning curves, and higher operating costs. This manuscript serves as a blueprint for building an outstanding RMVr program while preserving excellent results and improving operative efficiency.

A critical aspect of this study was the decision to maintain strict inclusion criteria in the initial cohort of patients. At the beginning of the study, the authors only included patients for whom a simple MVr was anticipated (i.e. single scallop prolapse). Importantly, this definition of MVr complexity is consistent with the ACC/AHA Guidelines for the Management of Patients with Valvular Heart Disease.[3] As their familiarity with the robotic platform improved over time, the authors gradually expanded their inclusion criteria to incorporate patients with more complex mitral pathologies (e.g. anterior/bileaflet/multi-scallop prolapses, extensive Barlow's disease). Importantly, all patients underwent successful RMVr without the need for mitral valve replacement.

The authors are to be commended for a comprehensive report of their initial experience with RMVr. Their obvious keys to success include teamwork, focus, and progression of case difficulty, with an unyielding 
standard of excellence.

References:

1. Fujita, T., et al., Benefits of robotically-assisted surgery for complex mitral valve repair. Interact Cardiovasc Thorac Surg, 2021.32 (3): p. 417-425.

2. Ramzy, D., et al., Complex robotic correction for complex degenerative mitral valve disease. Ann Cardiothorac Surg, 2017.6 (1): p. 70-72.

3. Otto, C.M., et al., 2020 ACC/AHA Guideline for the Management of Patients With Valvular Heart Disease: Executive Summary: A Report of the American College of Cardiology/American Heart Association Joint Committee on Clinical Practice Guidelines. Circulation, 2021.143 (5): p. e35-e71. 